\title{
Rapid mixing of the switch Markov chain for strongly stable degree sequences*
}

\author{
Georgios Amanatidis \\ University of Essex \\ Colchester, UK \\ University of Amsterdam \\ Amsterdam, The Netherlands \\ georgios.amanatidis@essex.ac.uk
}

\author{
Pieter Kleer \\ Max Planck Institute for Informatics \\ Saarbrücken, Germany \\ pkleer@mpi-inf.mpg.de
}

August 11, 2020

\begin{abstract}
The switch Markov chain has been extensively studied as the most natural Markov Chain Monte Carlo approach for sampling graphs with prescribed degree sequences. We show that the switch chain for sampling simple undirected graphs with a given degree sequence is rapidly mixing when the degree sequence is so-called strongly stable. Strong stability is satisfied by all degree sequences for which the switch chain was known to be rapidly mixing based on Sinclair's multicommodity flow method up until a recent manuscript of Erdôs et al. (2019). Our approach relies on an embedding argument, involving a Markov chain defined by Jerrum and Sinclair (1990). This results in a much shorter proof that unifies (almost) all the rapid mixing results for the switch chain in the literature, and extends them up to sharp characterizations of P-stable degree sequences. In particular, our work resolves an open problem posed by Greenhill and Sfragara (2017).
\end{abstract}

Keywords: Switch Markov chain; degree sequence; sampling; mixing time

\footnotetext{
*A preliminary version of this work appeared, as part of a larger work, in the Proceedings of the 30th Annual ACM-SIAM Symposium on Discrete Algorithms (SODA 2019) [1].
} 


\section{Introduction}

A classical result due to Erdôs and Gallai [18] characterizes when a sequence of non-negative integers $d_{1} \geq \cdots \geq d_{n}$ can be realized as the degree sequence of a simple undirected (labelled) graph on $n$ vertices, i.e., if there exists a realization of the sequence. The Erdós-Gallai theorem states that the sequence can be realized if and only if: the sum $d_{1}+\cdots+d_{n}$ is even and, for every $1 \leq k \leq n$,

$$
\sum_{i=1}^{k} d_{i} \leq k(k-1)+\sum_{i=k+1}^{n} \min \left\{d_{i}, k\right\} .
$$

Havel [27] and, independently, Hakimi [26], provide a simple polynomial-time algorithm to compute a realization if one exists. With the existence and construction problem being well-understood, in the last decades there has also been a great interest in uniformly sampling graphs with a given degree sequence, as it finds many applications, e.g., in hypothesis testing in network structures [38].

A prominent line of work for sampling graphs with given degrees is the Markov Chain Monte Carlo $(M C M C)$ method. Here one studies a random walk on the set of all realizations induced by a probabilistic algorithmic procedure that specifies how to make (small) random changes to the current realization. The probabilities with which realizations are turned into each other (arising from the algorithmic procedure) define a Markov chain on the set of all realizations. The idea, roughly, is that after a sufficient number of changes, the so-called mixing time of the Markov chain, the resulting realization corresponds to a sample from an almost uniform distribution over all realizations of the given degree sequence. The goal is to show that the chain mixes rapidly, meaning that one only needs to simulate the Markov chain for a polynomial (in the number of vertices $n$ ) number of steps in order to obtain an approximately uniform sample.

One of the most well-known probabilistic procedures for making these small changes uses local operations called switches (also known as swaps or transpositions); see Figure 1 for an example. The notion of a switch naturally gives rise to the switch algorithm: start with some initial realization $G_{0}$ with degree sequence $\boldsymbol{d}$, that can be computed in polynomial time using the Havel-Hakimi algorithm mentioned earlier, and repeatedly apply random switches. This can be done by selecting a tuple of four nodes $(x, y, v, w)$ uniformly at random, for instance. If, as in Figure 1, the edges $\{x, y\}$ and $\{v, w\}$ are present in $G_{0}$, and $\{x, w\}$ and $\{v, y\}$ are not, we switch the edges $\{x, y\}$ and $\{v, w\}$. Does this algorithm have all the desired properties? That is, do we have the guarantee that if one applies sufficiently many switches that the resulting graph is close to a uniform sample from the set of all realizations?

In order to establish the correctness of this approach we consider the switch Markov chain on the set of all realizations induced by this algorithmic procedure. The transition probabilities of the Markov chain are given by the probabilities with which the switches are applied. We should first check that this is an aperiodic, irreducible Markov chain with the uniform distribution as stationary distribution, i.e., that it actually does the job. Aperiodicity is easy to check, as well as the fact that the chain is reversible with respect to the uniform distribution. Irreducibility is less trivial, but still well-understood. The fact that every two realizations of a degree sequence $\boldsymbol{d}$ can be transformed into one another by a finite sequence of switches-which implies that the switch chain is irreducible-already follows from the works of Havel [27] and Hakimi [26], see also Petersen [39]; for a direct proof (in English), see Taylor [43].

It then follows that the switch algorithm is a fully polynomial almost uniform sampler if the switch Markov chain is rapidly mixing. The switch Markov chain has been shown to be rapidly mixing for various degree sequences $[6,24,25,37,16,14]$, but it is still open whether it is rapidly mixing for all degree sequences. 


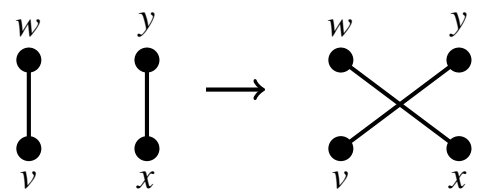

Figure 1: A switch in which edges $\{v, w\},\{x, y\}$ are replaced by $\{v, y\},\{x, w\}$. Note that the degree sequence is preserved when applying a switch operation.

\subsection{Our contributions}

In Section 3, we present a new proof idea for showing rapid mixing of the switch Markov chain that unifies and extends all ranges of degrees for which the switch chain was known to be rapidly mixing before the conference version of this work [1]. More recent follow-up work is given at the end of the related work subsection. In particular, we show that the switch chain is rapidly mixing for all strongly stable degree sequences (Theorem 3.1). We introduce strong stability as a stricter version of the notion of $P$-stability [30], which roughly means that the number of realizations that a degree sequence has, does not vary too much if the degree sequence is slightly perturbed. The strong stability condition is satisfied by the degree sequences in the works [32, 6, 24, 37, 16, 14] and by characterizations of $P$-stability [28]. In particular, our results resolve an open question posed by Greenhill [24] (see Corollary 3.2). We should note that the unification of the existing results mentioned so far is qualitative rather than quantitative, in the sense that our simpler, indirect approach provides weaker polynomial bounds for the mixing time. For examples of explicit mixing time bounds we refer the reader to $[6,7,25]$.

The proofs of the results in $[37,24,16,15]$ for the analysis of the switch Markov chain are all using conceptually similar ideas to the ones introduced by Cooper, Dyer and Greenhill [6] for the analysis of the switch chain for regular undirected graphs, and are based on the multi-commodity flow method of Sinclair [40]. Sinclair's method, roughly speaking, states that if one can define a good multi-commodity flow (of which the demands depend on the stationary distribution) in the state space graph of the Markov chain in which no edge gets too congested, then the Markov chain mixes rapidly. The individual parts of this method for the known switch chain analyses can become quite technical and require long proofs.

In this work we take a different approach for proving that the switch chain is rapidly mixing. First we analyze an easier auxiliary Markov chain introduced by Jerrum and Sinclair [30]; such a chain can be used to sample realizations that almost have a given fixed degree sequence. We show that there exists an efficient multi-commodity flow for the auxiliary chain when the given instance is strongly stable, and then show how it can be transformed into an efficient multi-commodity flow for the switch chain. In this last step we compare two Markov chains with different state spaces, as the auxiliary chain samples from a strictly larger set of graphs than the switch chain. For this part of the proof we rely on embedding arguments similar to those by Feder, Guetz, Mihail and Saberi [17].

\subsection{Related work}

Jerrum and Sinclair [30] provide a fully polynomial almost uniform sampler (FPAUS) for generating realizations of degree sequences coming from any P-stable family of sequences (see Section 2.1). Jerrum, Sinclair and Vigoda [31] give the first FPAUS for sampling bipartite graphs with any given degree sequence. This is a corollary of their breakthrough work [31] on approximating the permanent of a non-negative matrix. In the latter work the problem is reduced to that of sampling perfect matchings in a bipartite graph. Bezáková, Bhatnagar and Vigoda [3] provide a more direct and improved sampler of that in [31]. It is open whether or not there exists an FPAUS for general undirected degree sequences. More generally, it is 
still open if there is an FPAUS for sampling perfect matchings in undirected graphs. Recently Stefankovič, Wilmes and Vigoda [41] showed that the approach in [31] does not go through for general undirected graphs.

One drawback of the sampler of Jerrum and Sinclair [30] is that it works with auxiliary states. Kannan, Tetali and Vempala [32] introduce the switch chain as a simpler and more direct sampler that does not have to work with auxiliary states. They addressed the mixing time of such a switch-based Markov chain for the regular bipartite case. Cooper, Dyer and Greenhill [6] then gave a rapid mixing proof for regular undirected graphs, and later Greenhill [24] extended this result to certain ranges of irregular degree sequences; see also Greenhill and Sfragara [25]. Miklós, Erdôs and Soukup [37] proved rapid mixing for the half-regular bipartite case, and Erdős, Miklós and Toroczkai [16] for the almost half-regular case. Recently, Erdôs, Mezei and Miklós [14] presented a range of bipartite degree sequences unifying and generalizing the results in $[37,16]$. Carstens and Kleer [5] study a variant of the switch Markov chain for bipartite graphs, in which multiple switches are performed simultaneously, and show it is rapidly mixing if and only if the switch Markov chain is rapidly mixing.

Switch-based Markov chain Monte Carlo approaches have also been studied for other graph sampling problems. Feder et al. [17], as well as Cooper et al. [8], study the mixing time of a Markov chain using a switch-like probabilistic procedure (called a flip) for sampling connected graphs. For sampling perfect matchings, switch-based Markov chains have also been studied, see, e.g., the recent work of Dyer, Jerrum and Müller [10] and references therein.

Non-MCMC algorithms for sampling graphs with given degrees. There also exist graph sampling algorithms not relying on the MCMC method, many inspired by the configuration model [4]. Although this model in general outputs a loopy multigraph, there exist degree sequences for which the configuration model yields a simple graph with positive probability. For $d$-regular degree sequences, in which all nodes have degree $d \in \mathbb{N}$, the configuration model outputs a simple graph with probability roughly $e^{-(d-1)^{2} / 4}$. This is actually an exact uniform sample from the set of all simple $d$-regular graphs (not just close to a uniform sample). The inverse of the probability for obtaining a simple graph is bounded by a polynomial in $n$ if and only if $d=O(\sqrt{\log (n)})$.

Steger and Wormald [42] analyze a natural variant of the configuration model in which repeatedly only feasible edges are added uniformly at random. An edge is feasible if it does not create a loop or parallel edge. They show this procedure gives an output distribution over all simple $d$-regular graphs which is asymptotically uniform, for $d=o\left(n^{1 / 28}\right)$. This range was extended to $d=o\left(n^{1 / 3-\epsilon}\right)$ by Kim and $\mathrm{Vu}$ [33], and later by Bayati, Kim and Saberi [2] to $d=o\left(n^{1 / 2-\epsilon}\right)$. The latter work [2] also studies irregular degree sequences.

McKay and Wormald [35] considered a different extension of the configuration model, in which first a loopy multigraph is generated, after which loops and parallel edges are carefully switched out. This procedure provides an exact uniform sample, in particular for regular graphs with $d=o\left(n^{1 / 3}\right)$, and runs in expected polynomial time. Based on this algorithm, Gao and Wormald [22] provided an algorithm that gives an exact uniform sample for $d=o(\sqrt{n})$, and later obtained similar results for power-law degree sequences [21]. Very recently, Gao and Greenhill [19] obtained results along this line in which there is a set of forbidden edges, that cannot be used in any realization.

There is also a line of work on importance sampling methods that generate graph samples with given degrees that are statistically independent, see, e.g., [2,9] and references therein. These samples do not satisfy the properties that we require in this work, although in general they are sufficient to carry out certain statistical tests.

Follow-up work since the conference version [1]. Erdôs et al. [11] show, in a recent preprint, that the proof templates used in $[6,24,37,16,15]$ can be adjusted to show rapid mixing for $P$-stable degree sequences. This is an improvement over our result for strongly stable degree sequences. In particular, it allows the 
authors to claim rapid mixing of the switch Markov chain for certain power-law degree sequences. These sequences are claimed to be $P$-stable in [22], based on results in [20], but it is not known if they are also strongly stable (or provably non strongly stable) in general.

\subsection{Outline}

In Section 2 we give all the necessary Markov chain preliminaries and we formally describe the switch chain and the chain of Jerrum and Sinclair [30] that we use as an auxiliary Markov chain. In Section 3 we present our rapid mixing results for strongly stable degree sequences, as well as our new proof approach for the switch Markov chain.

\section{Preliminaries}

We begin with the necessary preliminaries regarding Markov chains and the multicommodity flow method of Sinclair [40]. For Markov chain definitions not given here, see, e.g., [34].

Let $\mathcal{M}=(\Omega, P)$ be an ergodic, time-reversible Markov chain over state space $\Omega$ with transition matrix $P$ and stationary distribution $\pi$. We write $P^{t}(x, \cdot)$ for the distribution over $\Omega$ at time step $t$ given that the initial state is $x \in \Omega$. The total variation distance at time $t$ with initial state $x$ is

$$
\Delta_{x}(t)=d_{T V}\left(P^{t}(x, \cdot), \pi\right)=\max _{S \subseteq \Omega}\left|P^{t}(x, S)-\pi(S)\right|=\frac{1}{2} \sum_{y \in \Omega}\left|P^{t}(x, y)-\pi(y)\right|,
$$

and the mixing time $\tau(\epsilon)$ is defined as

$$
\tau(\epsilon)=\max _{x \in \Omega}\left\{\min \left\{t: \Delta_{x}\left(t^{\prime}\right) \leq \epsilon \text { for all } t^{\prime} \geq t\right\}\right\} .
$$

Informally, $\tau(\epsilon)$ is the number of steps until the Markov chain is $\epsilon$-close to its stationary distribution independently of the initial state $x \in \Omega$. A Markov chain is said to be rapidly mixing if the mixing time can be upper bounded by a function polynomial in $\ln (|\Omega| / \epsilon)$.

It is well-known that, since the Markov chain is time-reversible, the matrix $P$ only has real eigenvalues $1=\lambda_{0}>\lambda_{1} \geq \lambda_{2} \geq \cdots \geq \lambda_{|\Omega|-1}>-1$. We may replace the transition matrix $P$ of the Markov chain by $(P+I) / 2$, to make the chain lazy, and hence guarantee that all its eigenvalues are non-negative. It then follows that the second-largest eigenvalue of $P$ is $\lambda_{1}$. In this work we always consider the lazy versions of the Markov chains involved. It follows directly from Proposition 1 in [40] that

$$
\tau(\epsilon) \leq \frac{1}{1-\lambda_{1}}\left(\ln \left(1 / \pi_{*}\right)+\ln (1 / \epsilon)\right),
$$

where $\pi_{*}=\min _{x \in \Omega} \pi(x)$. For the special case where $\pi$ is the uniform distribution, the above bound becomes

$$
\tau(\epsilon) \leq \frac{1}{1-\lambda_{1}}(\ln (|\Omega|)+\ln (1 / \epsilon)) .
$$

The quantity $\left(1-\lambda_{1}\right)^{-1}$ can be upper bounded using the multicommodity flow method of Sinclair [40].

We define the state space graph of the chain $\mathcal{M}$ as the directed graph $\boldsymbol{G}$ with node set $\Omega$ that contains exactly the edges $(x, y) \in \Omega \times \Omega$ for which $P(x, y)>0$ and $x \neq y$. Let $\mathcal{P}=\cup_{x \neq y} \mathcal{P}_{x y}$, where $\mathcal{P}_{x y}$ is the set of simple paths between $x$ and $y$ in the state space graph $\boldsymbol{G}$. A flow $f$ in $\Omega$ is a function $\mathcal{P} \rightarrow[0, \infty)$ satisfying $\sum_{p \in \mathcal{P}_{x y}} f(p)=\pi(x) \pi(y)$ for all $x, y \in \Omega, x \neq y$. The flow $f$ can be extended to a function on oriented edges of $\boldsymbol{G}$ by setting $f(e)=\sum_{p \in \mathcal{P}: e \in p} f(p)$, so that $f(e)$ is the total flow routed through $e \in E(\boldsymbol{G})$. 
Let $\ell(f)=\max _{p \in \mathcal{P}: f(p)>0}|p|$ be the length of a longest flow carrying path, and let $\rho(e)=f(e) / Q(e)$ be the load of the edge $e$, where $Q(e)=\pi(x) P(x, y)$ for $e=(x, y)$. The maximum load of the flow is $\rho(f)=\max _{e \in E(\boldsymbol{G})} \rho(e)$. Sinclair ([40], Corollary $\left.6^{\prime}\right)$ shows that

$$
\left(1-\lambda_{1}\right)^{-1} \leq \rho(f) \ell(f) .
$$

We use the following standard technique for bounding the maximum load of a flow in case the chain $\mathcal{M}$ has uniform stationary distribution $\pi$. Suppose $\theta$ is the smallest positive transition probability of the Markov chain between two distinct states. If $b$ is such that $f(e) \leq b /|\Omega|$ for all $e \in E(\boldsymbol{G})$, then it follows that $\rho(f) \leq b / \theta$. Thus, we have

$$
\tau(\epsilon) \leq \frac{\ell(f) \cdot b}{\theta} \ln (|\Omega| / \epsilon)
$$

Now, if $\ell(f), b$ and $1 / \theta$ can be bounded by a function polynomial in $\log (|\Omega|)$, it follows that the Markov chain $\mathcal{M}$ is rapidly mixing. In this case, we say that $f$ is an efficient flow. Note that in this approach the transition probabilities do not play a role as long as $1 / \theta$ is polynomially bounded.

\subsection{Graphic degree sequences and the switch chain}

A sequence of non-negative integers $\boldsymbol{d}=\left(d_{1}, \ldots, d_{n}\right)$ is called a graphic degree sequence if there exists a simple, undirected, labeled graph on $n$ nodes having degrees $d_{1}, \ldots, d_{n}$; such a graph is called a realization of $\boldsymbol{d}$. For a given degree sequence $\boldsymbol{d}, \mathcal{G}(\boldsymbol{d})$ denotes the set of all realizations of $\boldsymbol{d}$. Throughout this work we only consider sequences $\boldsymbol{d}$ with positive components, and for which $\mathcal{G}(\boldsymbol{d}) \neq \emptyset$. Let $\mathcal{G}^{\prime}(\boldsymbol{d})=\cup_{\boldsymbol{d}^{\prime}} \mathcal{G}\left(\boldsymbol{d}^{\prime}\right)$ with $d^{\prime}$ ranging over the set

$$
\left\{\boldsymbol{d}^{\prime}: d_{j}^{\prime} \leq d_{j} \text { for all } j \text {, and } \sum_{i=1}^{n}\left|d_{i}-d_{i}^{\prime}\right| \leq 2\right\} \text {. }
$$

That is, we have (i) $\boldsymbol{d}^{\prime}=\boldsymbol{d}$, or (ii) there exist distinct $\kappa, \lambda$ such that $d_{i}^{\prime}=d_{i}-1$ if $i \in\{\kappa, \lambda\}$ and $d_{i}^{\prime}=d_{i}$ otherwise, or (iii) there exists a $\kappa$ so that $d_{i}^{\prime}=d_{i}-2$ if $i=\kappa$ and $d_{i}^{\prime}=d_{i}$ otherwise. In the case (ii) we say that $\boldsymbol{d}^{\prime}$ has two nodes with degree deficit one, and in the case (iii) we say that $\boldsymbol{d}^{\prime}$ has one node with degree deficit two. An infinite family $\mathcal{D}$ of graphic degree sequences is called $P$-stable [30] if there exists a polynomial $q(n)$ such that for all $\boldsymbol{d} \in \mathcal{D}$ we have $\left|\mathcal{G}^{\prime}(\boldsymbol{d})\right| /|\mathcal{G}(\boldsymbol{d})| \leq q(n)$, where $n$ is the number of components of $\boldsymbol{d}$.

Jerrum and Sinclair [30] define the following Markov chain on $\mathcal{G}^{\prime}(\boldsymbol{d})$, which will henceforth be referred to as the $J S$ chain. ${ }^{1}$

Let $G \in \mathcal{G}^{\prime}(\boldsymbol{d})$ be the current state of the JS chain. Choose an ordered pair of vertices $(i, j)$ uniformly at random:

- If $G \in \mathcal{G}(\boldsymbol{d})$ and $(i, j)$ is an edge of $G$, delete $(i, j)$ from $G$ (Type 0 transition),

- If $G \notin \mathcal{G}(\boldsymbol{d})$ and the degree of $i$ in $G$ is less than $d_{i}$, and $(i, j)$ is not an edge of $G$, add $(i, j)$ to $G$; if this causes the degree of $j$ to exceed $d_{j}$, select an edge $(j, k)$ uniformly at random and delete it. (In case the degree of $j$ did not exceed $d_{j}$, we call this a Type 2 transition, otherwise we call it a Type 1 transition.)

In case the degree of $j$ does not exceed $d_{j}$ in the second case, we call this a Type 2 transition.

The graphs $G, G^{\prime} \in \mathcal{G}^{\prime}(\boldsymbol{d})$ are $J S$ adjacent if they are adjacent in the state space graph $\boldsymbol{G}_{\mathrm{JS}}$ of the JS chain, i.e., if $G$ can be obtained from $G^{\prime}$ with positive probability in one transition of the JS chain. The properties of the JS chain, stated in Theorem 2.1 below, are easy to check [30].

\footnotetext{
${ }^{1}$ A slightly different definition of stability is given by Jerrum, McKay and Sinclair [28]. Based on this variant, one could define the corresponding variant of the JS chain. Nevertheless, the definitions of stability in [28] and [30] (and their corresponding definitions of strong stability) are equivalent. To avoid confusion, here we only use the definitions in [30].
} 
Theorem 2.1. The JS chain is irreducible, aperiodic and symmetric, and, hence, has uniform stationary distribution over $\mathcal{G}^{\prime}(\boldsymbol{d})$. Moreover, $P\left(G, G^{\prime}\right)^{-1} \leq 2 n^{3}$ for all JS adjacent $G, G^{\prime} \in \mathcal{G}^{\prime}(\boldsymbol{d})$, and also the maximum in- and out-degrees in $\boldsymbol{G}_{\mathrm{JS}}$ are bounded by $n^{3}$.

We say that two graphs $G, G^{\prime}$ are within distance $r$ in the JS chain if there exists a path of at most length $r$ from $G$ to $G^{\prime}$ in $\boldsymbol{G}_{\mathrm{JS}}$. By $\operatorname{dist}(G, \boldsymbol{d})$ we denote the minimum distance of $G$ to any of the elements in $\mathcal{G}(\boldsymbol{d})$. The following parameter will play a central role in this work. Let

$$
k_{\mathrm{JS}}(\boldsymbol{d})=\max _{G \in \mathcal{G}^{\prime}(\boldsymbol{d})} \operatorname{dist}(G, \boldsymbol{d}) .
$$

Based on the parameter $k_{\mathrm{JS}}(\boldsymbol{d})$, we define the notion of strong stability. The simple observation in Proposition 2.3 justifies the terminology.

Definition 2.2 (Strong stability). An infinite family of graphic degree sequences $\mathcal{D}$ is called strongly stable if there exists a constant $\ell$ such that $k_{\mathrm{JS}}(\boldsymbol{d}) \leq \ell$ for all $\boldsymbol{d} \in \mathcal{D}$.

Proposition 2.3. If $\mathcal{D}$ is strongly stable, then it is P-stable.

Proof. Suppose $\mathcal{D}$ is strongly stable with respect to the constant $\ell$. Let $\boldsymbol{d} \in \mathcal{D}$ be a degree sequence with $n$ components. For every $G \in \mathcal{G}^{\prime}(\boldsymbol{d}) \backslash \mathcal{G}(\boldsymbol{d})$ choose some $\varphi(G) \in \mathcal{G}(\boldsymbol{d})$ within distance $k=k_{\mathrm{JS}}(\boldsymbol{d})$ of $G$. As the in-degree of any node in $\boldsymbol{G}_{\mathrm{JS}}$ is bounded by $n^{3}$, the number of paths with length at most $k$ that end up at any particular graph in $\mathcal{G}(\boldsymbol{d})$ is upper bounded by $\left(n^{3}\right)^{k}$. Therefore, $\left|\mathcal{G}^{\prime}(\boldsymbol{d})\right| /|\mathcal{G}(\boldsymbol{d})| \leq n^{3 k} \leq n^{3 \ell}$, meaning that $\mathcal{D}$ is $P$-stable, since $\ell$ is constant.

Finally, the lazy version of the switch chain on $\mathcal{G}(\boldsymbol{d})$ is defined as follows; see, e.g., [6].

Let $G \in \mathcal{G}(\boldsymbol{d})$ be the current state of the switch chain:

- With probability $1 / 2$, do nothing.

- Otherwise, attempt to perform a switch operation: select two node-disjoint edges $\{a, b\}$ and $\{x, y\}$ uniformly at random, and select a perfect matching $M$ on nodes $\{x, y, a, b\}$ uniformly at random (there are three possible options). If $M \cap E(G)=\emptyset$, then delete $\{a, b\},\{x, y\}$ from $E(G)$ and add the edges of $M$.

The graphs $G, G^{\prime} \in \mathcal{G}(\boldsymbol{d})$ are switch adjacentif they are adjacent in the state space graph $\boldsymbol{G}_{\text {sw }}$ of the switch chain, i.e., if $G$ can be obtained from $G^{\prime}$ with positive probability in one transition of the chain. Note that here this is equivalent to the condition $\left|E(G) \triangle E\left(G^{\prime}\right)\right|=4$. Below we summarize some properties of the switch chain; see, e.g., [25] and references therein. The bound on the transition probabilities follows from a simple counting argument.

Theorem 2.4. The switch chain is irreducible, aperiodic and symmetric, and, thus, has uniform stationary distribution over $\mathcal{G}(\boldsymbol{d})$. Also, we have $P\left(G, G^{\prime}\right)^{-1} \leq 6 n^{4}$ for all switch adjacent $G, G^{\prime} \in \mathcal{G}(\boldsymbol{d})$, and the maximum in- and out-degrees in $\boldsymbol{G}_{\mathrm{sw}}$ are bounded by $n^{4}$.

\section{Switch chain for strongly stable sequences}

Theorem 3.1, below, is our main result regarding the mixing time of the switch chain for strongly stable degree sequences. Its proof is divided in two parts. First, in Section 3.1, by giving an efficient multicommodity flow, we show that for any $\boldsymbol{d}$ in a family of strongly stable degree sequences the JS chain is rapidly mixing on $\mathcal{G}^{\prime}(\boldsymbol{d})$. Then, in Section 3.2, we show that such an efficient flow for the JS chain on $\mathcal{G}^{\prime}(\boldsymbol{d})$ can be transformed into an efficient flow for the switch chain on $\mathcal{G}(\boldsymbol{d})$. This yields the following theorem. 
Theorem 3.1. Let $\mathcal{D}$ be a strongly stable family of degree sequences with respect to some constant $k$. Then there exists a polynomial $q(n)$ such that, for any $0<\epsilon<1$, the mixing time $\tau_{\mathrm{sw}}$ of the switch chain for a graphic sequence $\boldsymbol{d}=\left(d_{1}, \ldots, d_{n}\right) \in \mathcal{D}$ satisfies

$$
\tau_{\mathrm{sw}}(\epsilon) \leq q(n)^{k} \ln (1 / \epsilon) .
$$

We next discuss a direct corollary of Theorem 3.1 which was posed as an open question in [24]. Its proof is essentially the same as that of a similar result for a slightly different, but equivalent, notion of stability in [28]. It is given here for self-containment.

Corollary 3.2. Let $\mathcal{D}=\mathcal{D}(\delta, \Delta)$ be the set of all graphic degree sequences $\boldsymbol{d}$ satisfying

$$
(\Delta-\delta+1)^{2} \leq 4 \delta(n-\Delta-1)
$$

where $\delta$ and $\Delta$ are the minimum and maximum component of $\boldsymbol{d}$, respectively. For any $\boldsymbol{d} \in \mathcal{D}$, we have $k_{\mathrm{JS}}(\boldsymbol{d}) \leq 6$. Hence, the switch chain is rapidly mixing for sequences in $\mathcal{D}$.

Proof. We first introduce some notation, using the same terminology as in [28]. Let $G=(V, E)$ be an undirected graph. For distinct $u, v \in V$ we say that $u, v$ are co-adjacent if $\{u, v\} \notin E$, and $\{u, v\}$ is called a non-edge. An alternating path of length $q$ in $G$ is a sequence of (not necessarily distinct) nodes $v_{0}, v_{1}, \ldots, v_{q}$ such that $\left\{v_{i}, v_{i+1}\right\}$ is an edge when $i$ is even, and a non-edge if $i$ is odd. The path is called a cycle if $v_{0}=v_{q}$. As in the proof of Theorem 2 in [28], we need the following lemma of Jerrum, McKay, and Sinclair [28].

Lemma 3.3 ([28]). Let $H$ be an undirected n-vertex graph with distinguished vertices $s$ and $t$ (not necessarily distinct), and suppose the set of vertices adjacent to $s$ is equal to the set of vertices adjacent to $t$. Suppose that $\delta_{\min }$ and $\delta_{\max }$ are natural numbers such that the degrees of all vertices other than $s$ and $t$ lie in the range $\left[\delta_{\min }, \delta_{\max }\right]$, and such that $s$ and themselves have degree at least $\delta_{\min }+1$. If $\left(\delta_{\max }-\delta_{\min }+1\right)^{2} \leq 4 \delta_{\min }\left(n-\delta_{\max }-1\right)$, then there exists an edge-disjoint alternating path in $G$ which starts at $s$, ends at $t$, and has length 1,3,5 or 7 .

Let $G \in \mathcal{G}^{\prime}(\boldsymbol{d}) \backslash \mathcal{G}(\boldsymbol{d})$. First consider the case where for the degree sequence $\boldsymbol{d}^{\prime}$ of $G$ there exist $x, y$ so that

$$
d_{i}^{\prime}= \begin{cases}d_{i}-1 & \text { if } i=x, y \\ d_{i} & \text { otherwise }\end{cases}
$$

If $\{x, y\}$ is a non-edge, then clearly $\operatorname{dist}(G, \boldsymbol{d})=1$, as we can then simply add the edge $\{x, y\}$ to obtain a graph in $\mathcal{G}(\boldsymbol{d})$. Therefore, assume that $\{x, y\}$ is an edge in $G$. It follows that both nodes $x$ and $y$ have degree at most $n-2$ in $G-\{x, y\}$, and therefore there exist two nodes $a$ and $b$ so that $\{x, a\}$ and $\{y, b\}$ are non-edges. If nodes $a$ and $b$ have the same set of neighbors in $G+\{x, a\}+\{y, b\}$, we can directly apply Lemma 3.3 to the graph $G+\{x, a\}+\{y, b\}$ to obtain an odd alternating path from $a$ to $b$ of length at most 7. Otherwise, without loss of generality, we may assume that there exists a node $c$ which is a neighbor of $a$ but not of $b$ (again in $G+\{x, a\}+\{y, b\}$ ). We can then remove $\{a, c\}$ and add $\{c, b\}$ in order to get a degree surplus of two at node $b$, and then we can apply Lemma 3.3 with $s=t=b$ in the graph $G+\{x, a\}+\{y, b\}-\{a, c\}+\{c, b\}$ (if $c=x$ this part of the proof can be skipped by choosing $a=b$ in the beginning of the argument, as both $\{x, b\}$ and $\{y, b\}$ are then non-edges in the graph $G$ we start with). In any case, it follows that there exists an alternating (between edges and non-edges of $G$ ) circuit of even length containing the edge $\{x, y\}$ in $G$ of length at most 12 . This implies that $\operatorname{dist}(G, \boldsymbol{d}) \leq 6$. That is, in at most six moves in the JS chain we can now reach an element in $\mathcal{G}(\boldsymbol{d})$.

Next, suppose that for the degree sequence $d^{\prime}$ of $G$ there exists some $x$ so that

$$
d_{i}^{\prime}= \begin{cases}d_{i}-2 & \text { if } i=x \\ d_{i} & \text { otherwise }\end{cases}
$$


It is clear that the degree of $x$ is at most $n-2$ in $G$. Let $\{x, a\}$ and $\{x, b\}$ be two non-edges. Applying similar steps as in the previous case to the graph $G+\{x, a\}+\{x, b\}$ it follows that $G$ has an alternating path of length at most 9 starting in $a$ and ending in $b$. It then again follows that $\operatorname{dist}(G, d) \leq 6$.

Explicit families satisfying these conditions are given in [28]. For instance, all sequences $\boldsymbol{d}$ with (i) $\delta(\boldsymbol{d}) \geq 1$ and $\Delta(\boldsymbol{d}) \leq 2 \sqrt{n}-2$, or (ii) $\delta(\boldsymbol{d}) \geq \frac{1}{4} n$ and $\Delta(\boldsymbol{d}) \leq \frac{3}{4} n-1$ satisfy (2). The bound in Corollary 3.2 is in a sense best possible with respect to the graph parameters involved. Namely, there exist non-stable degree sequence families the members of which only slightly violate (2); see the discussion in [28] for details.

\subsection{Flow for the Jerrum-Sinclair chain}

Jerrum and Sinclair [30] claim that, by using similar arguments as given in [29], the JS chain can be shown to be rapidly mixing for (some) families of stable degree sequences. For completeness, we prove in Theorem 3.4 that the chain is indeed rapidly mixing for any family of strongly stable degree sequences. We do so using the ideas introduced in [29] for sampling perfect matchings in dense graphs.

Theorem 3.4 ([30]). Let $\mathcal{D}$ be a strongly stable family of degree sequences. Then there exist polynomials $p(n)$ and $r(n)$ such that for any $\boldsymbol{d} \in \mathcal{D}$ there exists an efficient multicommodity flow $f$ for the JS chain on $\mathcal{G}^{\prime}(\boldsymbol{d})$ satisfying $\max _{e} f(e) \leq p(n) /\left|\mathcal{G}^{\prime}(\boldsymbol{d})\right|$ and $\ell(f) \leq r(n)$.

Our proof of Theorem 3.4, given below, uses conceptually similar arguments to the ones used in [6] for the analysis of the switch chain on regular undirected graphs. However, the analysis done here for the JS chain is, in our opinion, easier and cleaner than the corresponding analysis for the switch chain. In particular, the so-called circuit processing procedure is much simpler in our setting, as it only involves altering edges in the symmetric difference of two realizations in a straightforward fashion. In the switch chain analyses $[6,25,37,13,16,14]$ one also has to temporarily alter edges that are not in the symmetric difference and this significantly complicates things. Moreover, for the analysis of the JS chain, we can rely on arguments used (in a somewhat different context) by Jerrum and Sinclair [29] for the analysis of a Markov chain for sampling (near) perfect matchings of a given graph. This usage of arguments in [29] was, in fact, suggested by Jerrum and Sinclair [30] for showing that the JS chain is rapidly mixing for stable degree sequences.

We will use the following idea from [29]—used in a different setting —in order to restrict ourselves to establishing flow between states in $\mathcal{G}(\boldsymbol{d})$, rather than between all states in $\mathcal{G}^{\prime}(\boldsymbol{d})$. Assume that $\boldsymbol{d}$ is a degree sequence with $n$ components that is a member of a strongly stable family of degree sequences (with respect to some $k$ ).

Lemma 3.5. Let $f^{\prime}$ be a flow on $\boldsymbol{G}_{\mathrm{JS}}$ that routes $1 /\left|\mathcal{G}^{\prime}(\boldsymbol{d})\right|^{2}$ units of flow between any pair of states in $\mathcal{G}(\boldsymbol{d})$, so that $f^{\prime}(e) \leq b /\left|\mathcal{G}^{\prime}(\boldsymbol{d})\right|$ for all $e$ in $E\left(\boldsymbol{G}_{\mathrm{JS}}\right)$. Then $f^{\prime}$ can be extended to a flow $f$ on $\boldsymbol{G}_{\mathrm{JS}}$ that routes $1 /\left|\mathcal{G}^{\prime}(\boldsymbol{d})\right|^{2}$ units of flow between any pair of states in $\mathcal{G}^{\prime}(\boldsymbol{d})$ and has the property that for all e in $E\left(\boldsymbol{G}_{\mathrm{JS}}\right)$

$$
f(e) \leq q(n) \frac{b}{\left|\mathcal{G}^{\prime}(\boldsymbol{d})\right|},
$$

where $q(\cdot)$ is a polynomial whose degree only depends on $k_{\mathrm{JS}}(\boldsymbol{d})$. Moreover, $\ell(f) \leq \ell\left(f^{\prime}\right)+2 k_{\mathrm{JS}}(\boldsymbol{d})$.

Proof sketch. We describe how to extend the flow $f^{\prime}$ to $f$. For any $G \in \mathcal{G}^{\prime}(\boldsymbol{d}) \backslash \mathcal{G}(\boldsymbol{d})$ we fix some $\varphi(G) \in \mathcal{G}(\boldsymbol{d})$ within distance $k=k_{\mathrm{JS}}(\boldsymbol{d})$ of $G$, along with some simple path $p_{G}$ from $G$ to $\varphi(G)$ in $\boldsymbol{G}_{\mathrm{JS}}$ of length at most $k$. Note that, for all $G$, the existence of such a $\varphi(G)$ and of the corresponding path is guaranteed by the definition of $k$. Moreover, for $H \in \mathcal{G}(\boldsymbol{d})$, we define $\varphi(H)=H$.

The flow between $G, G^{\prime} \in \mathcal{G}^{\prime}(\boldsymbol{d})$ is now send as follows. First, if $G \neq \varphi(G)$, route $1 /\left|\mathcal{G}^{\prime}(\boldsymbol{d})\right|^{2}$ units of flow from $G$ to $\varphi(G)$ over $p_{G}$. Then use the flow-carrying paths used to send $1 /\left|\mathcal{G}^{\prime}(\boldsymbol{d})\right|^{2}$ units of flow 
between $\varphi(G)$ and $\varphi\left(G^{\prime}\right)$ in the flow $f^{\prime}$ (recall that multiple paths might be used for this in $f^{\prime}$ ). Finally, if $G^{\prime} \neq \varphi\left(G^{\prime}\right)$, use the reverse of $p_{G^{\prime}}$ to route $1 /\left|\mathcal{G}^{\prime}(\boldsymbol{d})\right|^{2}$ from $\varphi\left(G^{\prime}\right)$ to $G^{\prime}$. This fully defines the flow $f$, which is clearly an extension of $f^{\prime}$.

It is straightforward by the definition of $f$ that $\ell(f) \leq \ell\left(f^{\prime}\right)+2 k_{\mathrm{JS}}(\boldsymbol{d})$. It remains to show that for any edge $e$ of $\boldsymbol{G}_{\mathrm{JS}}, f(e)$ and $f^{\prime}(e)$ are polynomially related. For all $H \in \mathcal{G}(\boldsymbol{d})$, we have $\left|\varphi^{-1}(H)\right| \leq n^{3 k}$, as the in- and out-degrees of $\boldsymbol{G}_{\mathrm{JS}}$ are bounded by $n^{3}$ (Theorem 2.1). By using simple counting arguments, this fact has two important implications for an edge $e$ in $E\left(\boldsymbol{G}_{\mathrm{JS}}\right)$ :

$i$. Among the paths defined in the beginning of this proof, there are at most $k n^{3(k-1)}$ paths and at most $k n^{3(k-1)}$ "reverse" paths that could go through $e$. Note that each such path $p_{G}$ (resp. its reverse) is used at most $\left|\mathcal{G}^{\prime}(\boldsymbol{d})\right|$ times, once for each pair of the form $\left(G, G^{\prime}\right)$ (resp. of the form $\left(G^{\prime}, G\right)$ ), where $G^{\prime} \in \mathcal{G}^{\prime}(\boldsymbol{d})$.

ii. Any flow-carrying path of $f^{\prime}$ is used in $f$ at most $n^{6 k}$ times, carrying the same flow each time. So, the part of the total flow routed through $e$ under $f$ due to inflating $f^{\prime}$ cannot exceed the total flow routed through $e$ under $f^{\prime}$ by a factor larger than $n^{6 k}$.

Now combining the above two facts we get that, for any $e \in E\left(\boldsymbol{G}_{\mathrm{JS}}\right)$

$$
f(e) \leq \frac{2 k n^{3(k-1)}}{\left|\mathcal{G}^{\prime}(\boldsymbol{d})\right|}+n^{6 k} f^{\prime}(e) \leq 2 n^{6 k} \frac{b}{\left|\mathcal{G}^{\prime}(\boldsymbol{d})\right|},
$$

thus completing the proof.

We now continue with the proof of Theorem 3.4. It consists of four parts following, in a conceptual sense, the proof template in [6]. Whenever we refer to [29], the reader is referred to Section 3 of [29].

Proof of Theorem 3.4. We are going to define a flow $f^{\prime}$ on $\boldsymbol{G}_{\mathrm{JS}}$ as in Lemma 3.5. That is, we want $f^{\prime}$ to route $1 /\left|\mathcal{G}^{\prime}(\boldsymbol{d})\right|^{2}$ units of flow between any pair of states in $\mathcal{G}(\boldsymbol{d})$, so that $f^{\prime}(e) \leq b /\left|\mathcal{G}^{\prime}(\boldsymbol{d})\right|$ for any $e$ in $E\left(\boldsymbol{G}_{\mathrm{JS}}\right)$. Moreover, we are going to show that $b \leq p_{1}(n)$ and $\ell\left(f^{\prime}\right) \leq p_{2}(n)$ for some polynomials $p_{1}(\cdot), p_{2}(\cdot)$ whose degrees only depend on $k=k_{\mathrm{JS}}(\boldsymbol{d})$. In fact, we show that it suffices to use $p_{1}(n)=p_{2}(n)=n^{2}$.

Given such an $f^{\prime}$, the theorem follows from Lemma 3.5 and the fact that $\ln \left(\left|\mathcal{G}^{\prime}(\boldsymbol{d})\right|\right)$ is upper bounded by a polynomial in $n$. The latter follows from Equation (1) of McKay and Wormald [36] that implies that

$$
\left|\mathcal{G}\left(\boldsymbol{d}^{\prime}\right)\right| \leq n^{n^{2}}
$$

for any degree sequence $\boldsymbol{d}^{\prime}$ with $n$ components (see also [25]). So, by the definition of $\left|\mathcal{G}^{\prime}(\boldsymbol{d})\right|$ we have

$$
\left|\mathcal{G}^{\prime}(\boldsymbol{d})\right| \leq\left(\frac{n(n-1)}{2}+n+1\right) n^{n^{2}}
$$

and thus $\ln \left(\left|\mathcal{G}^{\prime}(\boldsymbol{d})\right|\right) \leq 3 n^{3}$.

Before we define $f^{\prime}$, we first introduce some basic terminology similar to that in [6]. Let $V$ be a set of labeled vertices, let $\prec_{E}$ be a fixed total order on the set $\{\{v, w\}: v, w \in V\}$ of edges, and let $\prec_{C}$ be a total order on all circuits on the complete graph $K_{V}$, i.e., $\prec_{C}$ is a total order on the closed walks in $K_{V}$ that visit every edge at most once. We fix for every circuit one of its vertices where the walk begins and ends. We emphasize that one can choose any orderings $\prec_{E}$ and $\prec_{C}$ here, which are not necessarily related nor induced by an ordering on the vertices.

For given $G, G^{\prime} \in \mathcal{G}(\boldsymbol{d})$, let $H=G \Delta G^{\prime}$ be their symmetric difference. We refer to the edges in $G \backslash G^{\prime}$ as blue, and the edges in $G^{\prime} \backslash G$ as red. A pairing of red and blue edges in $H$ is a bijective mapping that, for each node $v \in V$, maps every red edge adjacent to $v$, to a blue edge adjacent to $v$. The set of all pairings is denoted by $\Psi\left(G, G^{\prime}\right)$, and, with $\theta_{v}$ the number of red edges adjacent to $v$ (which is the same as the number of blue edges adjacent to $v$ ), we have $\left|\Psi\left(G, G^{\prime}\right)\right|=\Pi_{v \in V} \theta_{v}$ !. In what follows, it is convenient to slightly abuse this notation and write $\Psi\left(G \triangle G^{\prime}\right)$ rather than $\Psi\left(G, G^{\prime}\right)$. 


\subsubsection{Canonical paths and circuit processing}

Similar to the approach in [6], the goal is to construct for each pairing $\psi \in \Psi\left(G \triangle G^{\prime}\right)$ a canonical path from $G$ to $G^{\prime}$ that carries a $\left|\Psi\left(G \triangle G^{\prime}\right)\right|^{-1}$ fraction of the total flow from $G$ to $G^{\prime}$ in $f^{\prime}$. For notational convenience, for the remaining of the proof we write $u v$ instead of $\{u, v\}$ to denote an edge. For a given pairing $\psi$ and the total order $\prec_{E}$ given above, we first decompose $H$ into the edge-disjoint union of circuits in a canonical way. We start with the lexicographicly smallest edge $w_{0} w_{1}$ in $E_{H}$ and follow the pairing $\psi$ until we reach the edge $w_{k} w_{0}$ that was paired with $w_{0} w_{1}$. This defines the circuit $C_{1}$. If $C_{1}=E_{H}$, we are done. Otherwise, we pick the lexicographicly smallest edge in $H \backslash C_{1}$ and repeat this procedure. We continue generating circuits until $E_{H}=C_{1} \cup \cdots \cup C_{s}$. Note that all circuits have even length and alternate between red and blue edges, and that they are pairwise edge-disjoint. We form a path

$$
G=Z_{0}, Z_{1}, \ldots, Z_{M}=G^{\prime}
$$

from $G$ to $G^{\prime}$ in $\boldsymbol{G}_{\mathrm{JS}}$, by processing the circuits $C_{i}$ in turn according to the total order $<_{C}$. The processing of a circuit $C$ is the procedure during which all blue edges on $C$ are deleted, and all red edges of $C$ are added to the current realization, using the three types of transitions in the JS chain mentioned in Subsection 2.1. All other edges of the current realization remain unchanged. In general, this can be done similarly to the circuit processing procedure in [29].

Circuit processing [29]. Let $C=v x_{1} x_{2} \ldots x_{q} v$ be a circuit with start node $v$. We may assume, without loss of generality, that $v x_{1}$ is the lexicographicly smallest blue edge adjacent to the starting node $v$. We first perform a type 0 transition in which we remove the blue edge $v x_{1}$. Then we perform a sequence of $\frac{q-1}{2}$ type 1 transitions in which we add the red edge $x_{i} x_{i+1}$ and remove the blue edge $x_{i-1} x_{i}$ for $i=1,3, \ldots, q$. Finally we perform a type 2 transition in which we add the red edge $v x_{q}$. In particular, this means that the elements on the canonical path right before and after the processing of a circuit belong to $\mathcal{G}(\boldsymbol{d})$. It is easy to see that all the intermediate elements that we visit during the processing of the circuit $C$ belong to $\mathcal{G}^{\prime}(\boldsymbol{d}) \backslash \mathcal{G}(\boldsymbol{d})$, i.e., every element has either precisely two nodes with degree deficit one, or one node with degree deficit two. This is illustrated in Figures 3, 4 and 5 for the circuit in Figure 2.

For the next part, we define the notion of an encoding that can be used to bound the congestion of an edge in $\boldsymbol{G}_{\mathrm{JS}}$ using an injective mapping argument.

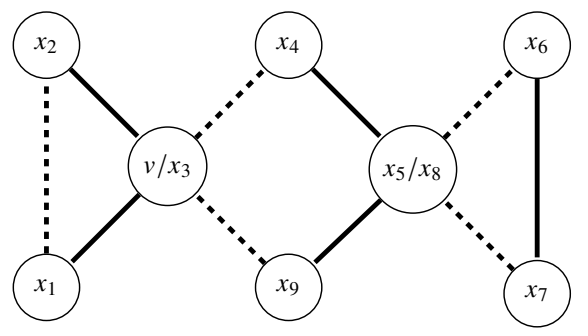

Figure 2: The circuit $C=v x_{1} x_{2} x_{3} x_{4} x_{5} x_{6} x_{7} x_{8} x_{9} v$ with $v=x_{3}$ and $x_{5}=x_{8}$. The blue edges are represented by the solid edges, and the red edges by the dashed edges.

\subsubsection{Encoding}

Let $t=\left(Z, Z^{\prime}\right)$ be a given transition of the Markov chain. Suppose two graphs $G$ and $G^{\prime}$ use the transition $t$ over some canonical path for some pairing $\psi \in \Psi\left(G \triangle G^{\prime}\right)$. Let $H=G \triangle G^{\prime}$. We define the encoding

$$
L_{t}\left(G, G^{\prime}\right)= \begin{cases}\left(H \triangle\left(Z \cup Z^{\prime}\right)\right)-e_{H, t} & \text { if } t \text { is a Type } 1 \text { transition, } \\ H \triangle\left(Z \cup Z^{\prime}\right) & \text { otherwise, }\end{cases}
$$



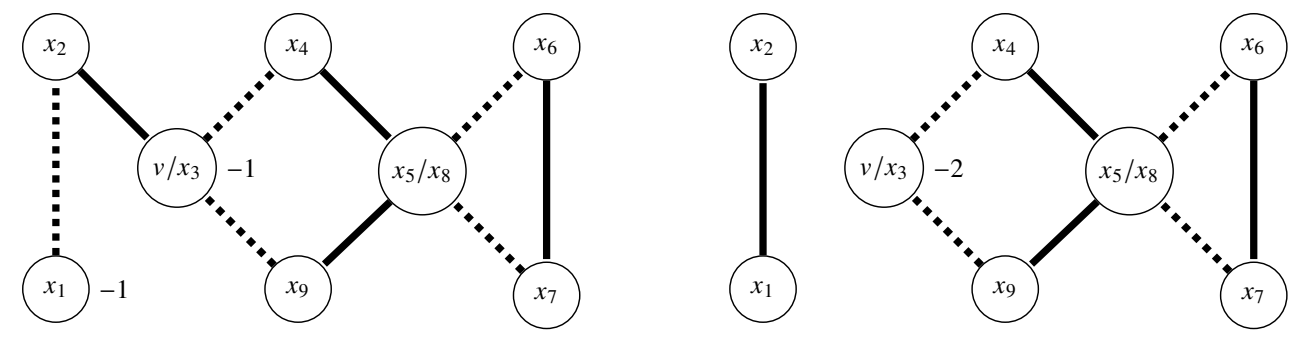

Figure 3: The edge $v x_{1}$ is removed using a Type 0 transition (left). The edge $x_{1} x_{2}$ is added and $x_{2} x_{3}=x_{2} v$ is removed using a Type 1 transition (right). We have also indicated the non-zero degree deficits.
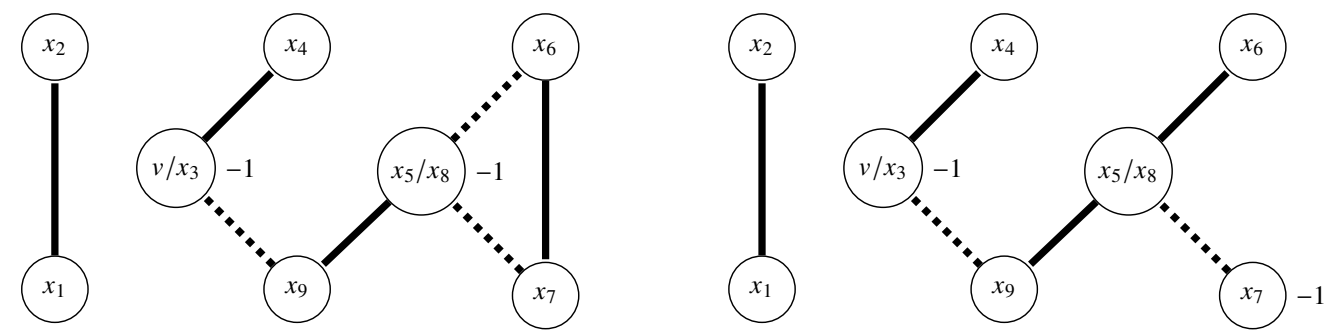

Figure 4: The edge $x_{3} x_{4}$ is added and $x_{4} x_{5}$ is removed using a Type 1 transition (left). The edge $x_{5} x_{6}$ is added and $x_{6} x_{7}$ is removed using a Type 1 transition (right).
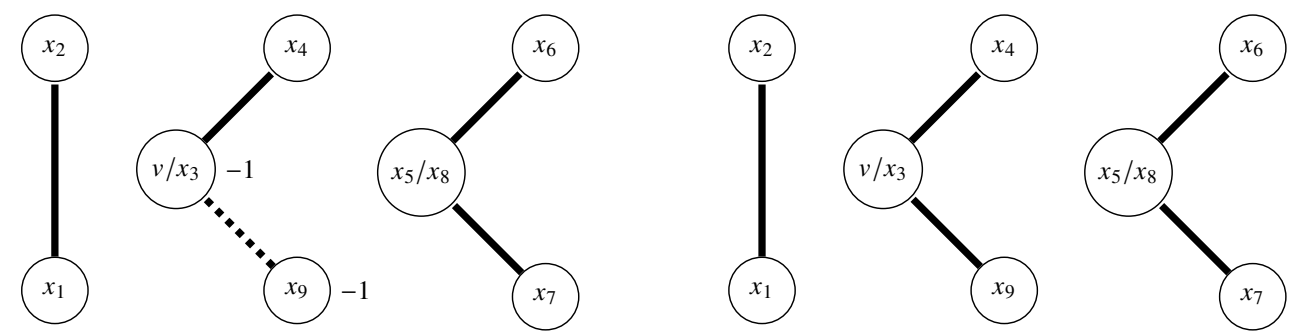

Figure 5: The edge $x_{7} x_{8}=x_{5} x_{8}$ is added and $x_{5} x_{9}=x_{8} x_{9}$ is removed using a Type 1 transition (left). The edge $v x_{9}$ is added using a Type 2 transition (right).

where $e_{H, t}$ is the first blue edge on the circuit that is currently being processed on the canonical path from $G$ to $G^{\prime}$ (for the given pairing $\psi$ ). This encoding is of a similar nature as the encoding used in [29]. An example is given in Figures 6,7 and 8. We also refer the reader to Figure 1 in [29] for a detailed example. ${ }^{2}$ The following lemma is crucial for the analysis.

Lemma 3.6. Given $t=\left(Z, Z^{\prime}\right), L$, and $\psi$, we can uniquely recover $G$ and $G^{\prime}$. That is, if $L$ is such that $L_{t}=L_{t}\left(G, G^{\prime}\right)$ for some pair $\left(G, G^{\prime}\right)$, then $\left(G, G^{\prime}\right)$ is the unique pair for which this is the case, given $t, L$, $\psi$.

Proof. We give the proof for when $t$ is a Type 1 transition. The cases of the two other types are similar, and arguably somewhat easier. The proof uses the arguments in [29] interpreted in our setting. First note

2 Although the perfect matching setting might seem different at first glance, it is actually closely related to our setting, with the only difference that the symmetric difference of two perfect matchings is the union of node-disjoint cycles, whereas in our setting the symmetric difference of two realizations is the union of edge-disjoint circuits. This is roughly why the notion of pairings is needed, as they allow us to uniquely determine the circuits. That is, the edge-disjoint circuits determined by the pairing are the analogue of the node-disjoint cycles in the perfect matching setting in [29]. 
that $L \Delta\left(Z \cup Z^{\prime}\right)$ is a graph in which there are precisely two nodes with odd degree. In particular, the edge $e_{H, t}$ is the unique edge (having as endpoints these odd degree nodes) that has to be added to $L \Delta\left(Z \cup Z^{\prime}\right)$ to obtain $H=G \triangle G^{\prime}$. That is, we have $\left(L \Delta\left(Z \cup Z^{\prime}\right)\right)+e_{H, t}=H$. The pairing $\psi$ then yields a unique circuit decomposition of $E(H)$ as explained at the beginning of the proof. From the transition $t$ it can be inferred which circuit is currently being processed, and, moreover, we can infer which edges of that circuit belong to $G$ and which to $G^{\prime}$. Furthermore, the global ordering $<_{C}$ on all circuits can then be used to determine for every other circuit whether it has been processed already or not. For every such circuit, we can then infer which edges on it belong to $G$ and which to $G^{\prime}$ by comparing with $Z$ (or $Z^{\prime}$ ). Therefore, $G$ and $G^{\prime}$ can be uniquely recovered from $t, L$ and $\psi$.
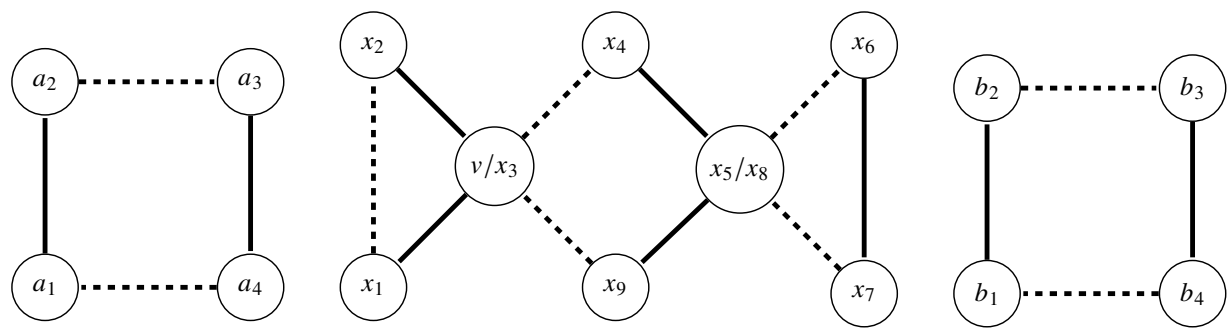

Figure 6: Symmetric difference $H=G \triangle G^{\prime}$ where the solid edges represent the edges $G$ and the dashed edges the edges of $G^{\prime}$. From left to right the circuit are numbered $C_{1}, C_{2}$ and $C_{3}$, and assume that this is also the order in which they are processed.

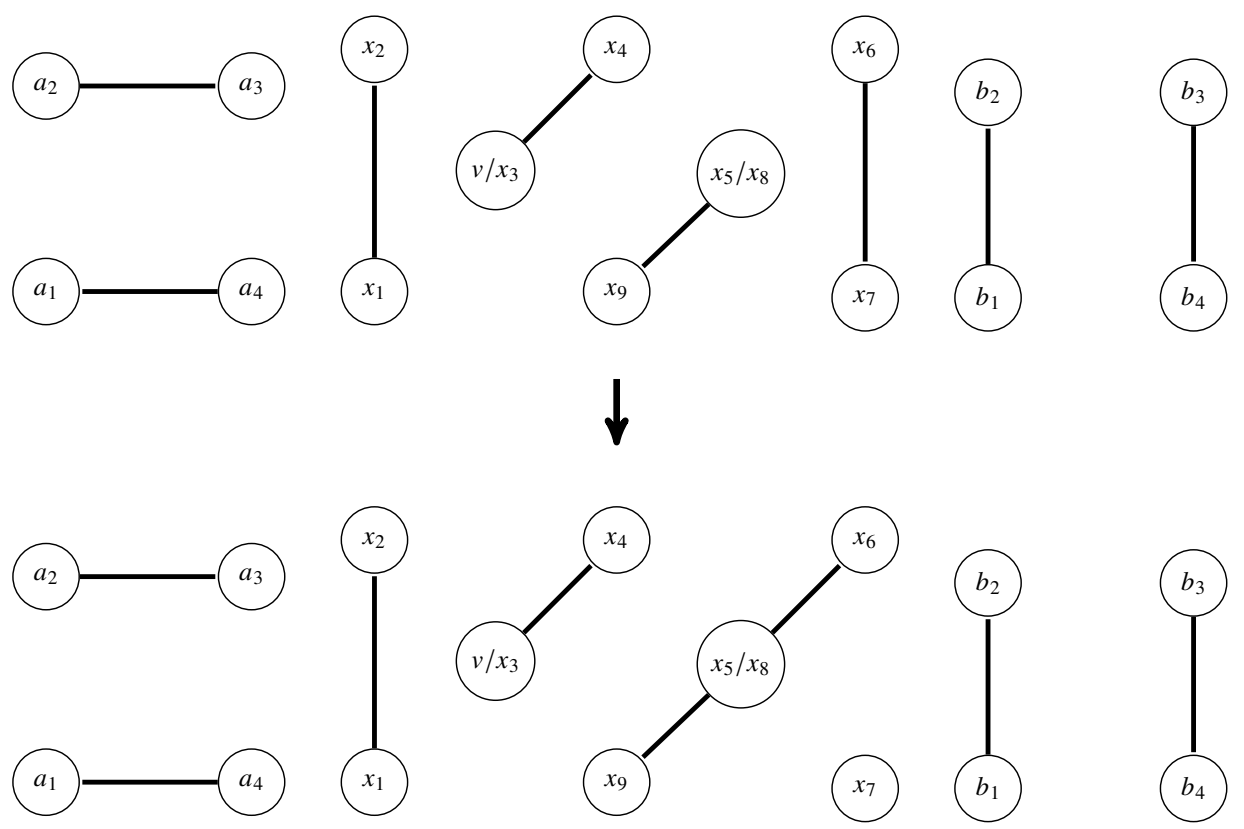

Figure 7: The transition $t=\left(Z, Z^{\prime}\right)$ that removes the edge $x_{6} x_{7}$ and adds the edge $x_{5} x_{6}$ as part of the processing of $C_{2}$. Note that $C_{1}$ has already been processed. The edges in $\left(E(G) \cup E\left(G^{\prime}\right)\right) \backslash E(H)$ are left out. 


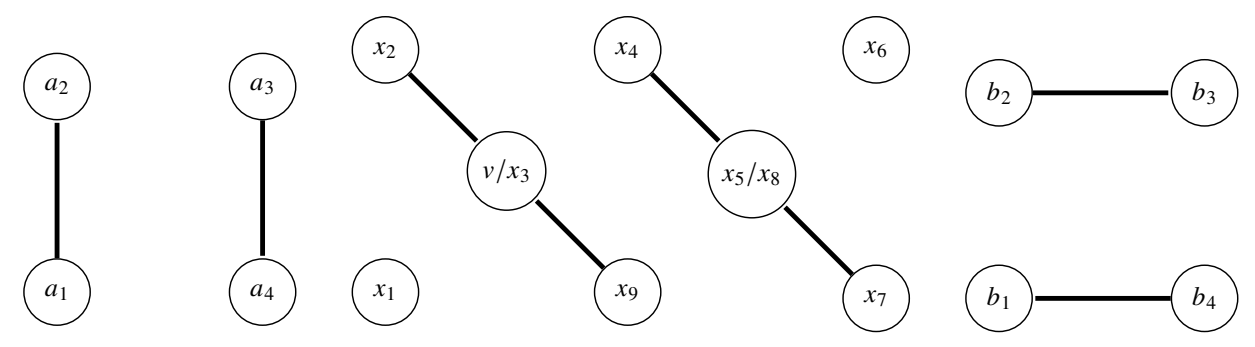

Figure 8: The encoding $L=L_{t}\left(G, G^{\prime}\right)$, where again the edges in $\left(E(G) \cup E\left(G^{\prime}\right)\right) \backslash E(H)$ are left out. Note that in this case $e_{H, t}=v x_{1}$ and that $L$ is itself an element of $\mathcal{G}^{\prime}(\boldsymbol{d})$.

\subsubsection{Bounding the congestion}

We complete the proof by using an injective mapping argument to bound the congestion of the flow $f^{\prime}$ on the edges of $\boldsymbol{G}_{\mathrm{JS}}$. The arguments used are a combination of ideas from [29] and the proof of Lemma 2.5 in [6] (see also Lemma 1 in [7]). We again focus on Type 1 transitions $t$ as the proofs for the other two types are similar but simpler.

For a tuple $\left(G, G^{\prime}, \psi\right)$, let $p_{\psi}\left(G, G^{\prime}\right)$ denote the canonical path from $G$ to $G^{\prime}$ for pairing $\psi$. Let

$$
\mathcal{L}_{t}=\left\{L_{t}\left(G, G^{\prime}\right) \mid\left(G, G^{\prime}, \psi\right) \in \mathcal{F}_{t}\right\}
$$

be the set of all (distinct) encodings $L_{t}$, where

$$
\mathcal{F}_{t}=\left\{\left(G, G^{\prime}, \psi\right): t \in p_{\psi}\left(G, G^{\prime}\right)\right\}
$$

is the set of all tuples $\left(G, G^{\prime}, \psi\right)$ such that the canonical path from $G$ to $G^{\prime}$ under pairing $\psi$ uses the transition $t$. A crucial observation is that every encoding $L_{t}\left(G, G^{\prime}\right)$ itself is an element of $\mathcal{G}^{\prime}(\boldsymbol{d})$ (see Figure 8 for an example). This implies that

$$
\left|\mathcal{L}_{t}\right| \leq\left|\mathcal{G}^{\prime}(\boldsymbol{d})\right|
$$

Moreover, with $H=G \triangle G^{\prime}$ and $L=L_{t}\left(G, G^{\prime}\right)$, the pairing $\psi$ has the property that it pairs up the edges of $E(H) \backslash E(L)$ and $E(H) \cap E(L)$ in such a way that for every node $v$ (with the exception of at most two nodes) each edge in $E(H) \backslash E(L)$ that is incident to $v$ is paired up with an edge in $E(H) \cap E(L)$ that is incident to $v$. However, there are either two nodes for which the incident edges in $E(H) \backslash E(L)$ exceed by 2 the incident edges in $E(H) \cap E(L)$, or one node for which the incident edges in $E(H) \backslash E(L)$ exceed by 4 the incident edges in $E(H) \cap E(L)$. These are exactly the two nodes with degree deficit 1 or the one node with degree deficit 2 in $L$; for the example in Figure 8 these are nodes $x_{1}$ and $x_{6}$. There $\psi$ pairs up each edge of $E(H) \cap E(L)$ to an edge of $E(H) \backslash E(L)$ but also two edges of $E(H) \backslash E(L)$ with each other; or in the case of one node with degree deficit 2, $\psi$ pairs up each edge of $E(H) \cap E(L)$ to an edge of $E(H) \backslash E(L)$ but also makes two pairs out of the remaining 4 edges in $E(H) \backslash E(L)$. Let $\Psi^{\prime}(L)$ be the set of all pairings with this property. ${ }^{3}$ Note that not every such pairing has to correspond to a tuple $\left(G, G^{\prime}, \psi\right)$ for which $t \in p_{\psi}\left(G, G^{\prime}\right)$.

By simply counting, we can upper bound $\left|\Psi^{\prime}(L)\right|$ in terms of $|\Psi(H)|$. We show the calculation for the case where $L$ has two nodes with degree deficit 1 . The case of one node with degree deficit 2 is very similar and the same upper bound works there as well. Suppose that $u, w$ are the two nodes of $L$ with degree deficit 1. Then

$$
\left|\Psi^{\prime}(L)\right|=\frac{\left(\theta_{u}+1\right) !}{2} \cdot \frac{\left(\theta_{w}+1\right) !}{2} \cdot \prod_{v \in V \backslash\{u, w\}} \theta_{v} !
$$

${ }^{3}$ Remember that we do not need to know $G$ and $G^{\prime}$ in order to determine the set $H$. It can be found based on $L$ and the transition $t=\left(Z, Z^{\prime}\right)$, as described in the proof of Lemma 3.6. 


$$
\begin{aligned}
& =\frac{\left(\theta_{u}+1\right)\left(\theta_{w}+1\right)}{4} \cdot|\Psi(H)| \\
& \leq n^{2} \cdot|\Psi(H)| .
\end{aligned}
$$

Recall that $f^{\prime}$ routes $1 /\left|\mathcal{G}^{\prime}(\boldsymbol{d})\right|^{2}$ units of flow between any pair of states in $\mathcal{G}(\boldsymbol{d})$ and that for any particular pair $\left(G, G^{\prime}\right)$ this flow is equally split among the $\left|\Psi\left(G \triangle G^{\prime}\right)\right|$ canonical paths defined in Subsection 3.1.1. Putting everything together, we have

$$
\begin{array}{rlrl}
\left|\mathcal{G}^{\prime}(\boldsymbol{d})\right|^{2} f^{\prime}(e) & =\sum_{\left(G, G^{\prime}\right)} \sum_{\psi \in \Psi\left(G \triangle G^{\prime}\right)} \mathbf{1}\left(e \in p_{\psi}\left(G \triangle G^{\prime}\right)\right)\left|\Psi\left(G \triangle G^{\prime}\right)\right|^{-1} \\
& \leq \sum_{L \in \mathcal{L}_{t}} \sum_{\psi^{\prime} \in \Psi^{\prime}(L)}\left|\Psi\left(G \triangle G^{\prime}\right)\right|^{-1} & & \text { (using Lemma 3.6) } \\
& \leq n^{2} \sum_{L \in \mathcal{L}_{t}} 1 & & \text { (using (4)) } \\
& \leq n^{2} \cdot\left|\mathcal{G}^{\prime}(\boldsymbol{d})\right| . & \text { (using (3)) }
\end{array}
$$

The usage of Lemma 3.6 for the first inequality works as follows. Every tuple $\left(G, G^{\prime}, \psi\right) \in \mathcal{F}_{t}$ with encoding $L_{t}\left(G, G^{\prime}\right)$ generates a unique tuple in $\left\{L_{t}\left(G, G^{\prime}\right)\right\} \times \Psi^{\prime}\left(L_{t}\left(G, G^{\prime}\right)\right)$. But since, by Lemma 3.6, we can uniquely recover $G$ and $G^{\prime}$ from $L, t$ and $\psi$, we have that $\sum_{L \in \mathcal{L}_{t}}\left|\{L\} \times \Psi^{\prime}(L)\right|=\sum_{L \in \mathcal{L}_{t}} \sum_{\psi^{\prime} \in \Psi^{\prime}(L)} 1$ is an upper bound on the number of canonical paths that use $t$.

By rearranging (5) we get the upper bound for $f^{\prime}$ required in Lemma 3.5. What is left to show is that $\ell\left(f^{\prime}\right)$ is not too large. This, however, is determined by the way we defined the canonical paths. It is easy to see that for any canonical path between any two graphs $G, G^{\prime} \in \mathcal{G}(\boldsymbol{d})$ has length at most $\frac{3}{4}\left|E\left(G \triangle G^{\prime}\right)\right|$ and, therefore, $\ell\left(f^{\prime}\right) \leq n^{2}$. This concludes the proof of Theorem 3.4.

\subsection{Flow transformation}

The main result of this section, Theorem 3.7, will allow us to utilize Theorem 3.4. While the statement may seem somewhat involved, essentially we show that, when $\boldsymbol{d}$ comes from a family of strongly stable degree sequences, an efficient multicommodity flow for the JS chain on $\mathcal{G}^{\prime}(\boldsymbol{d})$ can be transformed into an efficient multicommodity flow for the switch chain on $\mathcal{G}(\boldsymbol{d})$. In combination with Theorem 3.4 this implies that if $\mathcal{D}$ is strongly stable, then for any sequence in $\mathcal{D}$ there exists an efficient flow for the switch chain. Like in most parts of this work, for the sake of simplicity, we did not attempt to optimize the bounds in the proof of the theorem .

Theorem 3.7. Let $\mathcal{D}$ be a strongly stable family of degree sequences with respect to some constant $k$, and let $p(n)$ and $r(n)$ be polynomials such that for any $\boldsymbol{d} \in \mathcal{D}$ there exists an efficient multicommodity flow $f_{\boldsymbol{d}}$ for the $J S$ chain on $\mathcal{G}^{\prime}(\boldsymbol{d})$ with the properties that (i) $\ell(f) \leq r(n)$ and (ii) for every edge of $\boldsymbol{G}_{\mathrm{JS}}$, we have $f(e) \leq p(n) /\left|\mathcal{G}^{\prime}(\boldsymbol{d})\right|$.

Then there exists a polynomial $t(n)$ such that for all $\boldsymbol{d} \in \mathcal{D}$ there is a feasible multicommodity flow $g_{\boldsymbol{d}}$ for the switch chain on $\mathcal{G}(\boldsymbol{d})$ with the properties that (i) $\ell\left(g_{\boldsymbol{d}}\right) \leq 2 k \ell\left(f_{\boldsymbol{d}}\right)$, and (ii) for every edge e of $\boldsymbol{G}_{\mathrm{sw}}$, we have

$$
g_{\boldsymbol{d}}(e) \leq t(n)^{k} \frac{p(n)}{|\mathcal{G}(\boldsymbol{d})|} .
$$

Proof. Let $\boldsymbol{d} \in \mathcal{D}$. For simplicity we will write $f$ and $g$ instead of $f_{\boldsymbol{d}}$ and $g_{\boldsymbol{d}}$ respectively. We let $\mathcal{P}_{x y}$ refer to the set of simple paths between $x$ and $y$ in $\boldsymbol{G}_{\mathrm{JS}}$ (not those in $\boldsymbol{G}_{\mathrm{sw}}$ ). We first introduce some additional notation. 
For every pair $(x, y) \in \mathcal{G}^{\prime}(d) \times \mathcal{G}^{\prime}(\boldsymbol{d})$ with $x \neq y$, and for any $p \in \mathcal{P}_{x y}$, we write $\alpha(p)=f(p)\left|\mathcal{G}^{\prime}(\boldsymbol{d})\right|^{2}$. Recall that since the stationary distribution of the JS chain is uniform on $\mathcal{G}^{\prime}(\boldsymbol{d})$ we have $\sum_{p \in \mathcal{P}_{x y}} f(p)=$ $\left|\mathcal{G}^{\prime}(\boldsymbol{d})\right|^{-2}$. Thus, $\sum_{p \in \mathcal{P}_{x y}} \alpha(p)=1$. Moreover, we define $\alpha(e)=\sum_{p \in \mathcal{P}_{x y}: e \in p} \alpha(p)=f(e)\left|\mathcal{G}^{\prime}(\boldsymbol{d})\right|^{2}$.

Now, for every $G \in \mathcal{G}^{\prime}(\boldsymbol{d}) \backslash \mathcal{G}(\boldsymbol{d})$ choose some $\varphi(G) \in \mathcal{G}(\boldsymbol{d})$ that is within distance $k$ of $G$ in the JS chain, and take $\varphi(G)=G$ for $G \in \mathcal{G}(\boldsymbol{d})$. Based on the arguments in the proof of Proposition 2.3, it follows that for any $H \in \mathcal{G}(\boldsymbol{d})$,

$$
\left|\varphi^{-1}(H)\right| \leq n^{3 k},
$$

using that the maximum in-degree of any element in $\boldsymbol{G}_{\mathrm{JS}}$ is upper bounded by $n^{3}$. In particular, this implies that

$$
\frac{\left|\mathcal{G}^{\prime}(\boldsymbol{d})\right|}{|\mathcal{G}(\boldsymbol{d})|} \leq n^{3 k}
$$

Let the flow $h$ be defined as follows for any given pair $(x, y)$. If $(x, y) \in \mathcal{G}(\boldsymbol{d}) \times \mathcal{G}(\boldsymbol{d})$, take $h(p)=$ $\alpha(p) /|\mathcal{G}(\boldsymbol{d})|^{2}$ for all $p \in \mathcal{P}_{x y}$. If either $x$ or $y$ is not contained in $\mathcal{G}(\boldsymbol{d})$, take $h(p)=0$ for every $p \in \mathcal{P}_{x y}$. Note that $h$ is a multicommodity flow that routes $1 /|\mathcal{G}(\boldsymbol{d})|^{2}$ units of flow between any pair $(x, y) \in \mathcal{G}(\boldsymbol{d}) \times \mathcal{G}(\boldsymbol{d})$, and zero units of flow between any other pair of states in $\mathcal{G}^{\prime}(\boldsymbol{d})$.

Note that

$$
h(e) \leq \frac{\left|\mathcal{G}^{\prime}(\boldsymbol{d})\right|^{2}}{|\mathcal{G}(\boldsymbol{d})|^{2}} \cdot f(e) \leq \frac{\left|\mathcal{G}^{\prime}(\boldsymbol{d})\right|^{2}}{|\mathcal{G}(\boldsymbol{d})|^{2}} \frac{p(n)}{\left|\mathcal{G}^{\prime}(\boldsymbol{d})\right|}=\frac{p(n)}{|\mathcal{G}(\boldsymbol{d})|} \frac{\left|\mathcal{G}^{\prime}(\boldsymbol{d})\right|}{|\mathcal{G}(\boldsymbol{d})|} \leq n^{3 k} \cdot \frac{p(n)}{|\mathcal{G}(\boldsymbol{d})|},
$$

using the definition of $h$ in the first inequality, the assumption on $f$ in the second inequality, and the upper bound of (8) in the last one.

Next, we merge the "auxiliary states" in $\mathcal{G}^{\prime}(\boldsymbol{d}) \backslash \mathcal{G}(\boldsymbol{d})$, i.e., the states not reached by the switch chain, with the elements of $\mathcal{G}(\boldsymbol{d})$. Informally speaking, for every $H \in \mathcal{G}(\boldsymbol{d})$ we merge all the nodes in $\varphi^{-1}(H)$ into a supernode. Self-loops created in this process are removed, and parallel edges between states are merged into one edge that gets all the flow of the parallel edges. Formally, we consider the graph $\Gamma$ where $V(\Gamma)=\mathcal{G}(\boldsymbol{d})$ and $e=\left(H, H^{\prime}\right) \in E(\Gamma)$ if and only if $H$ and $H^{\prime}$ are switch adjacent or if there exist $G \in \varphi^{-1}(H)$ and $G^{\prime} \in \varphi^{-1}\left(H^{\prime}\right)$ such that $G$ and $G^{\prime}$ are JS adjacent. Moreover, for a given $h$-flow carrying path $\left(G_{1}, G_{2}, \ldots, G_{q}\right)=p \in \mathcal{P}_{x y}$, let $p_{\Gamma}^{\prime}=\left(\varphi\left(G_{1}\right), \varphi\left(G_{2}\right), \ldots, \varphi\left(G_{q}\right)\right)$ be the corresponding (possibly non-simple) directed path in $\Gamma$. Any self-loops and cycles can be removed from $p_{\Gamma}^{\prime}$ and let $p_{\Gamma}$ be the resulting simple path in $\Gamma$. Over $p_{\Gamma}$ we route $h_{\Gamma}\left(p_{\Gamma}\right)=h(p)$ units of flow. Note that $h_{\Gamma}$ is a flow that routes $1 /|\mathcal{G}(\boldsymbol{d})|^{2}$ units of flow between any pair of states $(x, y) \in \mathcal{G}(\boldsymbol{d}) \times \mathcal{G}(\boldsymbol{d})$ in the graph $\Gamma$ and that $\ell\left(h_{\Gamma}\right) \leq \ell(f)$. Furthermore, the flow $h_{\Gamma}$ on an edge $\left(H, H^{\prime}\right) \in E(\Gamma)$ is then bounded by

$$
h_{\Gamma}\left(H, H^{\prime}\right) \leq \sum_{\substack{\left(G, G^{\prime}\right) \in \varphi^{-1}(H) \times \varphi^{-1}\left(H^{\prime}\right) \\ G \text { and } G^{\prime} \text { are JS adjacent }}} h\left(G, G^{\prime}\right),
$$

where the inequality (instead of an equality) follows from the fact that when we map a path $p \in \mathcal{P}_{x y}$ to the corresponding path $p_{\Gamma}$, some edges of the intermediate path $p_{\Gamma}^{\prime}$ may be deleted. Using (7), it follows that $\left|\varphi^{-1}(H) \times \varphi^{-1}\left(H^{\prime}\right)\right| \leq n^{3 k} \cdot n^{3 k}=n^{6 k}$ and therefore, in combination with (9) and (10), we have that

$$
h_{\Gamma}(e) \leq n^{3 k} \cdot n^{6 k} \cdot \frac{p(n)}{|\mathcal{G}(\boldsymbol{d})|} .
$$

Now recall how $E(\Gamma)$ was defined. An edge $\left(H, H^{\prime}\right)$ might have been added because: (i) $H$ and $H^{\prime}$ are switch adjacent (we call these edges of $\Gamma$ legal), or (ii) $H$ and $H^{\prime}$ are not switch adjacent but there exist $G \in \varphi^{-1}(H)$ and $G^{\prime} \in \varphi^{-1}\left(H^{\prime}\right)$ that are JS adjacent (we call these edges of $\Gamma$ illegal). The final step of the proof consists of showing that the flow on every illegal edge in $E(\Gamma)$ can be rerouted over a "short" path 

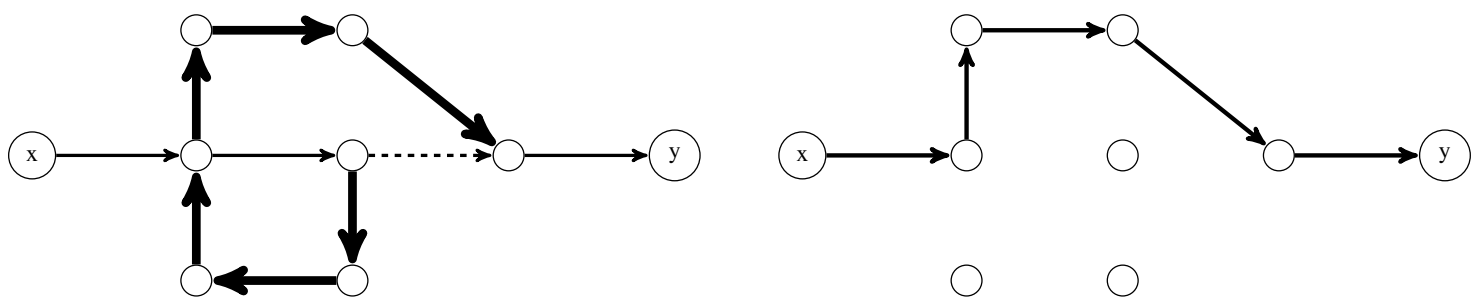

Figure 9: The dashed edge on the left represents an illegal edge, and the bold path represents a "short" detour. The shortcutted path on the right is the result of removing any loops and cycles.

consisting only of legal edges. In particular, for every flow carrying path $p$ using $e$, we are going to show that the flow $h_{\Gamma}(p)$ can rerouted over some legal detour, the length of which is bounded by a multiple of $k$. Doing this iteratively for every remaining illegal edge on $p$, we obtain a directed path $p^{\prime \prime}$ only using legal edges, i.e., edges of $\boldsymbol{G}_{\mathrm{sw}}$. Of course, $p^{\prime \prime}$ might not be simple, so any self-loops and cycles can be removed, as before, to obtain the simple legal path $p^{\prime}$. Figure 9 illustrates this procedure for a path with a single illegal edge. Note that deleting self-loops and cycles only decreases the amount of flow on an edge.

The crucial observation here is that if $\left(H, H^{\prime}\right) \in E(\Gamma)$, then $\left|E(H) \triangle E\left(H^{\prime}\right)\right| \leq 4 k$. That is, even though $H$ and $H^{\prime}$ might not be switch adjacent, they are not too far apart. To see this, first note that the symmetric difference of any two JS adjacent graphs has size at most 2. Moreover, if one of any two JS adjacent graphs is in $\mathcal{G}(\boldsymbol{d})$, then their symmetric difference has size 1 . In particular, for any $G^{*} \in \mathcal{G}^{\prime}(\boldsymbol{d})$, we have $\left|E\left(G^{*}\right) \triangle E\left(\varphi\left(G^{*}\right)\right)\right| \leq 2 k-1$.

Clearly, if $\left(H, H^{\prime}\right) \in E(\Gamma)$ is legal, then $\left|E(H) \triangle E\left(H^{\prime}\right)\right|=4 \leq 4 k$. Assume $\left(H, H^{\prime}\right) \in E(\Gamma)$ is illegal. Then there exist JS adjacent $G \in \varphi^{-1}(H)$ and $G^{\prime} \in \varphi^{-1}\left(H^{\prime}\right)$ and according to the above we have

$$
\begin{aligned}
\left|E(H) \triangle E\left(H^{\prime}\right)\right| & \leq|E(H) \triangle E(G)|+\left|E(G) \triangle E\left(G^{\prime}\right)\right|+\left|E\left(G^{\prime}\right) \triangle E\left(H^{\prime}\right)\right| \\
& \leq 2 k-1+2+2 k-1 \leq 4 k .
\end{aligned}
$$

Moreover, this implies that we can go from $H$ to $H^{\prime}$ in a "small" number of moves in the switch chain. This easily follows from most results showing that $\boldsymbol{G}_{\mathrm{sw}}$ is connected. Specifically, here we use the following result of Erdôs, Király, and Miklós [12] which implies that we can go from $H$ to $H^{\prime}$ in $2 k$ switches.

Theorem 3.8 (follows from Theorem 3.6 in [12]). Let $\boldsymbol{d}$ be a degree sequence. For any two graphs $H, H^{\prime} \in \mathcal{G}(\boldsymbol{d}), H$ can be transformed into $H^{\prime}$ using at most $\frac{1}{2}\left|E(H) \triangle E\left(H^{\prime}\right)\right|$ switches.

For every illegal edge $e \in E(\Gamma)$, we choose such a (simple) path from $H$ to $H^{\prime}$ with at most $2 k$ transitions and reroute the flow of $e$ over this path. Note that for any legal edge $e \in E(\Gamma)$, the number of illegal edge detours that use $e$ for this rerouting procedure, is at most $\left(n^{4}\right)^{2 k} \cdot\left(n^{4}\right)^{2 k}=n^{16 k}$, using the fact that in the state space graph of the switch chain the maximum degree of an element is at most $n^{4}$ and any illegal edge using $e$ in its rerouting procedure must lie within distance $2 k$ of $e$. Combining this with (11), we see that the resulting flow, $g$, satisfies

$$
g(e) \leq \frac{p(n) \cdot n^{9 k}+p(n) \cdot n^{16 k}}{|\mathcal{G}(\boldsymbol{d})|} .
$$

Note that $\ell(g) \leq 2 k \ell\left(h_{\Gamma}\right)$. This holds because every illegal edge on a flow-carrying path gives rise to at most $2 k$ additional edges as a result of rerouting the flow over legal edges, and the removal of loops and cycles from any resulting non-simple path can only decrease its length. Combining this inequality with $\ell\left(h_{\Gamma}\right) \leq \ell(f)$ (as we noted above), we get $\ell(g) \leq 2 k \ell(f)$. This completes the proof of (6), as we have now constructed a feasible multicommodity flow $g$ on $\boldsymbol{G}_{\mathrm{sw}}$ with the desired properties.

This also completes the proof of Theorem 3.1. 
Remark 3.9. Looking back, Lemma 3.5 is not needed for proving Theorem 3.1. Careful consideration of the proof of Theorem 3.7 shows that we can only focus on flow between states in $\mathcal{G}(\boldsymbol{d})$, since the flow $h$ given therein only has positive flow between states corresponding to elements in $\mathcal{G}(\boldsymbol{d})$. That is, when defining the flow $h$, we essentially forget about all flow in $f$ between any pair of states where at least one state is an auxiliary state, i.e., an element of $\mathcal{G}^{\prime}(\boldsymbol{d}) \backslash \mathcal{G}(\boldsymbol{d})$. Said differently, in Theorem 3.7 we could start with the assumption that $f$ routes $1 /\left|\mathcal{G}^{\prime}(\boldsymbol{d})\right|^{2}$ units of flow between any pair of states in $\mathcal{G}(\boldsymbol{d})$ in $\boldsymbol{G}_{\mathrm{JS}}$, and then the transformation still works. However, the formulations of Theorems 3.4 and 3.7 are more natural for describing a comparison between the JS and switch chains. In particular, Lemma 3.5 is needed to formally prove that the JS chain is rapidly mixing for strongly stable degree sequences.

\section{Conclusion}

We have shown that the switch Markov chain is rapidly mixing for the class of strongly stable degree sequences by using a novel proof approach for this problem. We believe that our ideas introduced in Section 3 can be also used to simplify the switch chain analyses in settings where there is some given forbidden edge set, the elements of which cannot be used in any (bipartite) realization [23, 25, 13, 15]. This is an interesting direction for future work, as it captures the case of sampling directed graphs. Further, it is not clear whether there exist degree sequence families that are $P$-stable but not strongly stable. For instance, in a recent work by Gao and Wormald [21], who provide a very efficient non-MCMC approximate sampler for certain power-law degree sequences, it is argued that these power-law degree sequences are $P$-stable. Is it the case these sequences are strongly stable as well? A central open question is how to go beyond (strong) stability.

\section{Acknowledgments}

This work was funded by NWO Gravitation project NETWORKS, Grant Number 024.002.00.

\section{References}

[1] Georgios Amanatidis and Pieter Kleer. Rapid mixing of the switch Markov chain for strongly stable degree sequences and 2-class joint degree matrices. In Proceedings of the 30th Annual ACM-SIAM Symposium on Discrete Algorithms, SODA 2019, pages 966-985, 2019.

[2] Mohsen Bayati, Jeong Han Kim, and Amin Saberi. A sequential algorithm for generating random graphs. Algorithmica, 58(4):860-910, 2010.

[3] Ivona Bezáková, Nayantara Bhatnagar, and Eric Vigoda. Sampling binary contingency tables with a greedy start. Random Structures \& Algorithms, 30(1-2):168-205, 2007.

[4] Béla Bollobás. A probabilistic proof of an asymptotic formula for the number of labelled regular graphs. European Journal of Combinatorics, 1(4):311-316, 1980.

[5] Corrie Jacobien Carstens and Pieter Kleer. Speeding up switch Markov chains for sampling bipartite graphs with given degree sequence. In Approximation, Randomization, and Combinatorial Optimization. Algorithms and Techniques, APPROX/RANDOM 2018, volume 116 of LIPIcs, pages 36:1-36:18, 2018.

[6] Colin Cooper, Martin E. Dyer, and Catherine S. Greenhill. Sampling regular graphs and a peer-to-peer network. Combinatorics, Probability \& Computing, 16(4):557-593, 2007.

[7] Colin Cooper, Martin E. Dyer, and Catherine S. Greenhill. Corrigendum: Sampling regular graphs and a peer-to-peer network. CoRR, abs/1203.6111, 2012. 
[8] Colin Cooper, Martin E. Dyer, Catherine S. Greenhill, and Andrew J. Handley. The flip Markov chain for connected regular graphs. Discrete Applied Mathematics, 254:56-79, 2018.

[9] Charo I. Del Genio, Hyunju Kim, Zoltán Toroczkai, and Kevin E. Bassler. Efficient and exact sampling of simple graphs with given arbitrary degree sequence. PLOS ONE, 5(4):1-7, 2010.

[10] Martin Dyer, Mark Jerrum, and Haiko Müller. On the switch Markov chain for perfect matchings. Journal of the $A C M, 64(2): 12: 1-12: 33,2017$.

[11] Péter L. Erdős, Catherine S. Greenhill, Tamás Róbert Mezei, István Miklós, Dániel Soltész, and Lajos Soukup. The mixing time of the swap (switch) Markov chains: a unified approach. CoRR, abs/1903.06600, 2019.

[12] Péter L Erdős, Zoltán Király, and István Miklós. On the swap-distances of different realizations of a graphical degree sequence. Combinatorics, Probability \& Computing, 22(3):366-383, 2013.

[13] Péter L. Erdôs, Sándor Z. Kiss, István Miklós, and Lajos Soukup. Approximate counting of graphical realizations. PLOS ONE, 10(7):1-20, 2015.

[14] Péter L. Erdős, Tamás Róbert Mezei, and István Miklós. Efficiently sampling the realizations of irregular, but linearly bounded bipartite and directed degree sequences. CoRR, abs/1712.01709, 2017.

[15] Péter L. Erdôs, Tamás Róbert Mezei, István Miklós, and Dániel Soltész. Efficiently sampling the realizations of bounded, irregular degree sequences of bipartite and directed graphs. PLOS ONE, 13(8):1-20, 082018.

[16] Péter L Erdôs, István Miklós, and Zoltán Toroczkai. A decomposition based proof for fast mixing of a Markov chain over balanced realizations of a joint degree matrix. SIAM Journal on Discrete Mathematics, 29(1):481-499, 2015 .

[17] Tomás Feder, Adam Guetz, Milena Mihail, and Amin Saberi. A local switch Markov chain on given degree graphs with application in connectivity of peer-to-peer networks. In Proceedings of the 47th Annual IEEE Symposium on Foundations of Computer Science, FOCS 2006, pages 69-76, 2006.

[18] Tibor Gallai and Paul Erdos. Graphs with prescribed degree of vertices. Lapok, 11:264-274, 1960.

[19] Pu Gao and Catherine S. Greenhill. Uniform generation of spanning regular subgraphs of a dense graph. CoRR, abs/1807.00964, 2018.

[20] Pu Gao and Nicholas Wormald. Enumeration of graphs with a heavy-tailed degree sequence. Advances in Mathematics, 287:412-450, 2016.

[21] Pu Gao and Nicholas C. Wormald. Uniform generation of random graphs with power-law degree sequences. In Proceedings of the 29th Annual ACM-SIAM Symposium on Discrete Algorithms, SODA 2018, pages 1741-1758, 2018.

[22] Pu Gao and Nilocas C. Wormald. Uniform generation of random regular graphs. SIAM Journal of Computing, 46(4):1395-1427, 2017.

[23] Catherine S. Greenhill. A polynomial bound on the mixing time of a Markov chain for sampling regular directed graphs. Electronic Journal of Combinatorics, 18(1), 2011.

[24] Catherine S. Greenhill. The switch Markov chain for sampling irregular graphs. In Proceedings of the 26th Annual ACM-SIAM Symposium on Discrete Algorithms, SODA 2015, pages 1564-1572, 2015.

[25] Catherine S. Greenhill and Matteo Sfragara. The switch Markov chain for sampling irregular graphs and digraphs. Theoretical Computer Science, 719:1-20, 2018.

[26] S. Hakimi. On realizability of a set of integers as degrees of the vertices of a linear graph. I. Journal of the Society for Industrial and Applied Mathematics, 10(3):496-506, 1962.

[27] Václav Havel. A remark on the existence of finite graphs. C̆asopis pro pěstování matematiky, 080(4):477-480, 1955.

[28] Mark Jerrum, Brendan D. McKay, and Alistair Sinclair. When is a graphical sequence stable? Random Graphs, Vol. 2, pages 101-116, 1992. 
[29] Mark Jerrum and Alistair Sinclair. Approximating the permanent. SIAM Journal on Computing, 18(6):1149-1178, 1989.

[30] Mark Jerrum and Alistair Sinclair. Fast uniform generation of regular graphs. Theoretical Computer Science, 73(1):91-100, 1990.

[31] Mark Jerrum, Alistair Sinclair, and Eric Vigoda. A polynomial-time approximation algorithm for the permanent of a matrix with nonnegative entries. Journal of the ACM, 51(4):671-697, 2004.

[32] Ravi Kannan, Prasad Tetali, and Santosh Vempala. Simple Markov-chain algorithms for generating bipartite graphs and tournaments. Random Structures and Algorithms, 14(4):293-308, 1999.

[33] J. H. Kim and V. H. Vu. Generating random regular graphs. Combinatorica, 26(6):683-708, 2006.

[34] David Asher Levin, Yuval Peres, and Elizabeth Lee Wilmer. Markov chains and mixing times. American Mathematical Society, 2009.

[35] Brendan D McKay and Nicholas C Wormald. Uniform generation of random regular graphs of moderate degree. Journal of Algorithms, 11(1):52-67, 1990.

[36] Brendan D. McKay and Nicholas C. Wormald. Asymptotic enumeration by degree sequence of graphs with degrees $o\left(n^{1 / 2}\right)$. Combinatorica, 11(4):369-382, 1991.

[37] István Miklós, Péter L. Erdôs, and Lajos Soukup. Towards random uniform sampling of bipartite graphs with given degree sequence. Electronic Journal of Combinatorics, 20(1), 2013.

[38] Benjamin P. Olding and Patrick J. Wolfe. Inference for graphs and networks: Adapting classical tools to modern data. In Data Analysis for Network Cyber-Security, pages 1-31, 2014.

[39] Julius Petersen. Die theorie der regulären graphs. Acta Mathematica, 15:193-220, 1891.

[40] Alistair Sinclair. Improved bounds for mixing rates of Markov chains and multicommodity flow. Combinatorics, Probability \& Computing, 1:351-370, 1992.

[41] Daniel Štefankovič, Eric Vigoda, and John Wilmes. On counting perfect matchings in general graphs. In Proceedings of the 13th Latin American Symposium on Theoretical Informatics, LATIN 2018, pages 873-885, 2018.

[42] A. Steger and N. C. Wormald. Generating random regular graphs quickly. Combinatorics, Probability \& Computing, 8(4):377-396, 1999.

[43] Richard Taylor. Constrained switchings in graphs. In Proceedings of the 8th Australian Conference on Combinatorial Mathematics, pages 314-336, 1981. 